Bull. Soc. math. France

129 (3), 2001, p. 339-356

\title{
TOWARDS A MORI THEORY ON COMPACT KÄHLER THREEFOLDS III
}

\author{
By Thomas Peternell
}

\begin{abstract}
Based on the results of the first two parts to this paper, we prove that the canonical bundle of a minimal Kähler threefold (i.e. $K_{X}$ is nef) is good, i.e. its Kodaira dimension equals the numerical Kodaira dimension, (in particular some multiple of $K_{X}$ is generated by global sections); unless $X$ is simple. "Simple" means that there is no compact subvariety through the very general point of $X$ and $X$ not Kummer. Moreover we show that a compact Kähler threefold with only terminal singularities whose canonical bundle is not nef, admits a contraction unless $X$ is simple with Kodaira dimension $-\infty$.

RÉSumÉ (À propos d'une théorie de Mori sur les variétés compactes kählériennes de dimension 3, III

Utilisant les résultats de la première et de la deuxième partie de ce travail, nous considérons des variétiés kählériennes minimales $X$ de dimension 3, i.e. dont le fibré canonique $K_{X}$ est nef. Alors $K_{X}$ est un fibré «good», i.e. dont la dimension de Kodaira est égale à la dimension de Kodaira numérique, sous l'exception possible que $X$ est simple, (i.e. il n'existe pas une sous-variété compacte contenant un points très general) et $X$ non Kummer. Le deuxième théorème dit que les variétés kählériennes $X$ de dimension 3 avec des singularités terminales de sorte que $K_{X}$ n'est pas nef, ont des contractions de Mori.
\end{abstract}

Texte reçu le 8 novembre 1999, révisé le 25 septembre 2000

Thomas Peternell, Mathematisches Institut der Universität, D-95445 Bayreuth (Germany) E-mail : thomas.peternell@uni-bayreuth.de

2000 Mathematics Subject Classification. - 32J17, 32Q15.

Key words and phrases. — Kähler threefolds, abundance, rational curves, Kodaira dimension. 


\section{Introduction}

In this note we continue the study of the bimeromorphic geometry of compact Kähler threefolds. The final aim should be - as in the algebraic case - to construct minimal models for threefolds with non-negative Kodaira dimension, describe the way how to get a minimal model, to prove abundance for the minimal models, i.e. semi-ampleness of the canonical bundle, and finally to construction Fano fibrations on appropriate models of threefolds with $\kappa=-\infty$.

Concerning abundance we show that the canonical divisor of a minimal Kähler threefold $X$ is good, i.e. $\kappa(X)=\nu(X)$, where $\nu(X)$ denotes the numerical Kodaira dimension, i.e. the largest number $m$ such that $K_{X}^{m} \not \equiv 0$; unless $X$ is simple and non-Kummer. Here $X$ is simple, if there is no positive-dimensional subvariety through the very general point of $X$ and $X$ is Kummer if it has a bimeromorphic model which is the quotient of a torus by a finite group. These simple non-Kummer varieties are expected not to exist but this can be only a consequence of a completely developped minimal model theory in the Kähler case. By [22], it follows from $\kappa(X)=\nu(X)$ that $K_{X}$ is semi-ample, i.e. some multiple $m K_{X}$ is generated by global sections. So abundance holds on Kähler threefolds with the possible exception of simple non-Kummer threefolds.

Furthermore we prove, using essentially Part 1 [1] and Part 2 [23] to this paper that a smooth compact Kähler threefold $X$ with $K_{X}$ not nef carries a contraction unless possibly $X$ is simple and non-Kummer. The main steps in the proof are the following:

1) Construction of some curve $C \subset X$ with $K_{X} \cdot C<0$. We distinguish the case $\kappa(X) \geq 0$ and $\kappa(X)=-\infty$. In the first case we examine carefully a member in the linear system $\left|m K_{X}\right|$ to construct $C$, in the second we use a result of a recent joint paper of Campana and the author saying that $X$ is uniruled unless $X$ is simple. In that sedond case it is immediately clear that we can choose $C$ rational.

2) Next we make $C$ rational (this step works for all compact Kähler threefolds). Here we construct from $C$ a non-splitting family of irreducible curve and examine its structure. The reason why this family exists is the deformation lemma of Ein-Kollár: a curve $C$ in a smooth threefold with $K_{X} \cdot C<0$ moves.

3) The last step is the construction of a contraction from a rational curve $C$ with $K_{X} \cdot C<0$. This was in large parts already done in [1] and [23]; here we finish the study in sect. 1 of this paper.

We summarise the results of this paper in the following two theorems.

THEOREM 1. - Let $X$ be a minimal Kähler threefold $(\mathbb{Q}$-factorial with at most terminal singularities). Assume that $X$ is not both simple and non-Kummer. Then $\kappa(X)=\nu(X)$, hence $K_{X}$ is semi-ample. In particular, if $\kappa(X)=0$

TOME $129-2001-\mathrm{N}^{\mathrm{O}} 3$ 
(and $X$ not simple non-Kummer), then $K_{X} \equiv 0$ and $m K_{X}=\mathcal{O}_{X}$ for some positive $m$.

TheOREM 2. - Let $X$ be a smooth compact Kähler threefold with $K_{X}$ not nef. Then $X$ carries a contraction unless (possibly) $X$ is simple with $\kappa(X)=-\infty$.

By a contraction we mean a surjective map $\varphi: X \rightarrow Y$ to a normal compact variety with connected fibers such that $-K_{X}$ is $\varphi$-ample and $b_{2}(X)=b_{2}(Y)+1$. We would like to have that $Y$ is again a Kähler space but at the moment we are still in trouble if $\varphi$ is the blow-up of a smooth curve in the smooth threefold $Y$ - in that case $Y$ will be only Kähler if $\varphi$ is choosen appropriately, namely the ray generated by the curves contracted by $\varphi$ must be extremal in the dual cone to the Kähler cone of $X$ (see [23]).

There are several problems arising.

(a) First of all we would like to contruct a curve with $K_{X} \cdot C<0$ also in the "simple" case if $K_{X}$ is not nef. This would mean that we should construct "directly" - i.e. without using any specific information on $X$ some curve $C$ with $K_{X} \cdot C<0$. This requires certainly new techniques and probably a threefold proof would work in any dimension and also with terminal singularities.

(b) We need to prove the existence of contractions also for $\mathbb{Q}$-Gorenstein threefolds with terminal singularities in order to perform the Mori program. The Gorenstein case will probably be the same as the smooth case, but in the presence of non-Gorenstein singularities there obstructions to moving curves so that some new arguments are needed.

(c) We must overcome the difficulty with the Kähler property of $Y$ in case of a blow-up $\varphi: X \rightarrow Y$ along a smooth curve.

As already observed in [23], one major consequence of this programme would be that simple Kähler threefolds are Kummer, in particular there are no simple threefolds of negative Kodaira dimension, all those being uniruled.

In the appendix we prove that Kummer threefolds $T / G$ with algebraic dimension 0 have Kodaira dimension 0 . This was already used in [23] and [2] but no proof was given.

I want to thank C. Okonek for interesting discussions on contact manifolds and the referee for very helpful remarks.

\section{Preliminaries}

0.1. - Let $X$ be a compact complex manifold and $L$ a line bundle on $X$. Fix a positive $(1,1)$-form $\omega$ on $X$. Then $L$ is nef if for every $\epsilon>0$ there exists a hermitian metric $h_{\epsilon}$ on $L$ with curvature $\Theta_{h_{\epsilon}} \geq-\epsilon \omega$. Since $X$ is compact, this notion does not depend on the choice of $\omega$. If $X$ is projective, we obtain the "old" definition, that $L \cdot C \geq 0$ for all irreducible compact curves $C \subset X$. For details we refer to [4]. 
Let $X$ be an irreducible normal compact complex in class $\mathcal{C}$, e.g. a normal compact Kähler space. Let $L$ be a line bundle on $X$. Then $L$ is nef if there is a desingularisation $\pi: \hat{X} \rightarrow X$ such that $\pi^{*}(L)$ is nef. By $[23,4.6]$ this is independent on the choice of $\pi$ at least in dimension 3 .

0.2. - A normal compact Kähler threefold ( $n$-fold) is minimal if $X$ is $\mathbb{Q}$ factorial, has only terminal singularities and $K_{X}$ (i.e. some $m K_{X}$ ) is nef.

0.3. - We will often use $C_{n, m}$ for Kähler threefolds: if $f: X \rightarrow Y$ is a surjective fiber space with $X$ a smooth compact Kähler threefold (so $n=3$ ) then $\kappa(X) \geq \kappa(Y)+\kappa(F)$, where $F$ denotes the general fiber $(m=\operatorname{dim} Y)$. See [7], [10], [27].

0.4. - A compact manifold is simple if there is no positive dimensional subvarieties through the very general point of $X$. The only known Kähler examples arise from tori. To make this more precise one says that a compact manifold (or normal compact space) is Kummer if $X$ is bimeromorphic to a quotient $T / G$ of a torus by a finite group $G$. The conjecture is that simple Kähler manifolds are Kummer. A standard reference here is [6].

0.5. - Some further notations: $a(X)$ denotes always the algebraic dimension of $X$. The irregularity of $X$ is $q(X)=\operatorname{dim} H^{1}\left(X, \mathcal{O}_{X}\right)$. Finally $N_{1}(X) \subset$ $H_{2}(X, \mathbb{R})$ is the vector space generated by the classes of irreducible curve. Inside $N_{1}(X)$ we have the closed cone $\overline{N E}(X)$ generated by the classes of irreducible curves.

\section{Abundance for Kähler threefolds}

In this section we prove the Abundance Conjecture for (non-simple) Kähler threefolds. First we show

Theorem 1.1. - Let $X$ be a minimal Kähler threefold with $\kappa(X)=0$. Assume that $X$ is not both simple and non-Kummer. Then $K_{X} \equiv 0$.

Proof. - The assertion being known for projective minimal threefolds by Miyaoka [19] and Kawamata [13], we shall assume that $X$ is non-projective. Then by [2] $X$ has a bimeromorphic model $X^{\prime}$ with at most quotient singularities such that there is a finite cover $Y \rightarrow X^{\prime}$, étale in codimension 1, with $Y$ a torus or a product $E \times S$ of an elliptic curve $E$ with a K3-surface $S$. In the algebraic case such a conclusion is of course false: Calabi-Yau threefolds are simply connected. The reason why the non-algebraic case is somehow more special than the projective is the existence of holomorphic 2-forms on non-algebraic threefolds (Kodaira's theorem).

Now back in our specific situation, we conclude that $K_{X^{\prime}} \equiv 0$. We claim that $X^{\prime}$ has only canonical singularities (a priori we know only that $\left(X^{\prime}, 0\right)$ has

TOME $129-2001-\mathrm{N}^{\mathrm{O}} 3$ 
$\log$ terminal singularities). This is seen as follows. Choose $m>0$ such that $m K_{X^{\prime}} \simeq \mathcal{O}_{X^{\prime}}$. Let $\pi: \hat{X} \rightarrow X^{\prime}$ be a desingularisation. Write

$$
m K_{\hat{X}}=\pi^{*}\left(m K_{X^{\prime}}\right)+\sum a_{i} E_{i}
$$

with $E_{i}$ the exceptional components of $\pi$. We need to show that $a_{i} \geq 0$ for all $i$. If some $a_{i}<0$, then $\pi_{*}\left(m K_{\hat{X}}\right)$ is a proper subsheaf of $m K_{X^{\prime}}=\mathcal{O}_{X^{\prime}}$; hence $H^{0}\left(m K_{\hat{X}}\right)=0$. Passing to a high multiple of $m$, we deduce $\kappa(\hat{X})=-\infty$, a contradiction.

So $X^{\prime}$ has only canonical singularities. Take a partial crepant resolution $\pi: \tilde{X} \rightarrow X^{\prime}$ [24]. One can even assure that $\tilde{X}$ is $\mathbb{Q}$-factorial [25], [12]. So $\tilde{X}$ has only terminal singularities and still $K_{\tilde{X}} \equiv 0$. Now the bimeromorphic meromorphic map $X \rightarrow \tilde{X}$ is an isomorphism in codimension 1 [8], [16]. Here we use the fact that $K_{X}$ is nef! Thus $K_{X} \equiv 0$.

REMARK 1.2. - In case $q(X)>0$, it is much easier to conclude; the argument being independent of [2]. Here is the reasoning in that case. Let $\alpha: X \rightarrow A$ be the Albanese map of $X$. Since $\kappa(X)=0, \alpha$ has to be surjective (by $C_{3,1}$ and $C_{3,2}$ ). Let $f: X \rightarrow Y$ be the Stein factorisation of $\alpha$ (we shall see that actually $\alpha$ has connected fibers). Notice that $\kappa(F)=0$ for the general fiber $F$ of $f$.

1) Suppose $\operatorname{dim} A=\operatorname{dim} Y=3$. Then $X \rightarrow A$ is unramified in codimension 1 , in fact, otherwise $K_{X}$ would contain the ramification divisor $R$ whose image in $A$ is a nef divisor with $\kappa>0$, so that $\kappa(R)>0$, hence $\kappa(X)>0$. Of course, this is well-known, at least in the algebraic case, see [14]. So $\alpha=f$ (by the universal property of $\alpha$ ), hence $\alpha$ is birational. Therefore

$$
K_{X}=\alpha^{*}\left(K_{A}\right)+\sum_{i \in I} \lambda_{i} E_{i}=\sum \lambda_{i} E_{i}
$$

where $E_{i}$ are the exceptional components of $\alpha$ and $\lambda_{i}>0$. Then $K_{X}$ being nef, it follows easily that $I=\varnothing$. So $K_{X}=\mathcal{O}_{X}$.

2) Next suppose that $\operatorname{dim} Y=1$. Hence $Y=A$ is an elliptic curve and again $f=\alpha$. Since $K_{F}$ is nef and $\kappa(F)=0$, we deduce that $K_{F}$ is torsion. Choose $m>0$ such that $m K_{X}$ is Cartier and that $h^{0}\left(m K_{X}\right)=1$. In particular $m K_{F}=\mathcal{O}_{F}$. Let $s \in H^{0}\left(m K_{X}\right)$ be a non-zero section. Then $s \mid F=0$ or $s \mid F$ has no zeroes. Writing

$$
m K_{X}=\sum a_{i} D_{i}
$$

with $a_{i}>0$, we conclude that $D_{i} \cap F=\varnothing$, i.e. $\operatorname{dim} D_{i}=0$. On the other hand $\sum a_{i} D_{i}$ is $\alpha$-nef, and this is only possible if some multiple $k m K_{X}$ is a multiple of fibers so that

$$
k m K_{X}=\alpha^{*}(L)
$$

BULLETIN DE LA SOCIÉtÉ MATHÉMATIQUE DE FRANCE 
with a line bundle $L$ on $A$. Since $\kappa(X)=0$, we have $\kappa(L)=0$, therefore $L=\mathcal{O}_{A}$.

3) Finally, let $\operatorname{dim} Y=2$. Again we conclude $f=\alpha$ and $Y=A$.

3.1) $a(A)=0$. Letting $s$ and $m K_{X}=\sum a_{i} E_{i}$ be as in 2), we again have $\operatorname{dim} \alpha\left(D_{i}\right) \leq 1$ (now $F$ is an elliptic curve). Since $A$ does not contain compact curves, we have $\operatorname{dim} \alpha\left(D_{i}\right)=0$ for all $i$, which again contradicts the nefness of $K_{X}$.

3.2) $a(A)=1$. Then we use the algebraic reduction $A \rightarrow B$ to an elliptic curve and obtain a map $g: X \rightarrow B$. Now we argue as in 2).

3.3) $a(A)=2$. By $C_{3,2}^{+}(c p$. [27]) we have $\operatorname{Var}(f)=0$. Hence there exists a finite cover $\tilde{A} \rightarrow A$ and a meromorphic generically finite map $F \times \tilde{A} \rightarrow X$ with an elliptic curve $F$. Thus $X$ is algebraic, contradiction. So this subcase cannot happen.

Corollary 1.3. - Let $X$ be a minimal Kähler threefold. Assume that $X$ is not both simple and non-Kummer. Then $\kappa(X)=\nu(X)$ and therefore $K_{X}$ is semi-ample.

Proof. - Again we may assume $X$ not projective. By [22] it suffices to prove $\kappa(X)=\nu(X)$.

1) We have $\kappa(X) \geq 0$ by the main result in [2] and the appendix.

2) If $\kappa(X)=0$, then $\nu(X)=0$ by (1.1).

3) If $\kappa(X) \geq 1$, we can apply $[11,7.3]$ to obtain $\kappa(X)=\nu(X)$; the arguments there remain valid in the Kähler case (at least for threefolds). Note that $K_{X}^{3}>0$ forces $X$ to be projective.

REMARK 1.4. - 1) The case that $X$ is simple and not Kummer remains open. The first difficulty is that we might have $K_{X}$ nef but $\kappa(X)=-\infty$. Furthermore, if $\kappa(X)=0$, then at least $\nu(X) \neq 1$. In case $X$ is projective, this is proved in [19]; his arguments work in the Kähler case as well. However it might still be possible that there is a simple non-Kummer minimal threefold with $\kappa(X)=0$ and $\nu(X)=2$.

Everything would be settled if one could produce a 1-form on a minimal simple threefold, possibly after finite cover, étale in codimension 1 or 2 . In fact, suppose that $q(X)>0$ and $X$ smooth for simplicity and later reference. Then, $X$ being simple, we must have $q(X)=3$, the Albanese $\alpha: X \rightarrow A$ has to be onto the threedimensional torus $A$. Since $A$ has no divisors, $\alpha$ is unramified in codimension 1, so that actually, arguing as in 1.1), $\alpha$ is bimeromorphic and $X$ is Kummer.

2) We treat one of the open problems in (1) in a special case. Assume that $X$ is smooth and that $m K_{X}=\mathcal{O}_{X}(D)$ with a smooth surface (and $X$ simple, $K_{X}$ nef). By adunction $K_{D}$ is nef and $K_{D}^{2}=0$. Therefore $c_{2}(D) \geq 0$. On the other

TOME $129-2001-\mathrm{N}^{\mathrm{O}} 3$ 
hand, $c_{2}(X) \cdot D=c_{2}(D)$ and $c_{2}(X) \cdot D=c_{2}(X) \cdot m K_{X}=-24 m \chi\left(X, \mathcal{O}_{X}\right)$. If $q(X)=0$, then $\chi\left(X, \mathcal{O}_{X}\right)>0$, hence $c_{2}(D)<0$, contradiction.

THEOREM 1.5. - Let $X$ be a smooth compact Kähler threefold with $\kappa(X)=0$. Suppose that $X$ is not both simple and non-Kummer. Then $X$ has a minimal model.

Proof. - The algebraic case being settled by Mori [21] (and previously by Ueno and Viehweg if $q(X)>0$, see [28]), we may assume that $X$ is not projective. Then however such a model has already been constructed in the proof of Theorem 1.1 (pass to $X^{\prime}$ as in 1.1 and then to $\tilde{X}$ in the notation of the proof of 1.1).

\section{Existence of contractions}

2.1. - Let $X$ be a smooth compact Kähler threefold and $C \subset X$ a rational curve such that $K_{X} \cdot C<0$. Then $C$ determines a non-splitting family of rational curves [1].

A non-splitting family of curves is by definition a family $\left(C_{t}\right)_{t \in T}$ parametrised by a compact irreducible variety such that all $C_{t}$ are irreducible reduced curves.

In [1] and [23] we proved that a non-splitting family $\left(C_{t}\right)_{t \in T}$ of rational curves defines a contraction $\varphi: X \rightarrow Y$ except in one case. This case is the following, called Case (E) below:

$X$ is non-projective, of course, $K_{X} \cdot C_{t}=-1$, the family $\left(C_{t}\right)$ fills up a non-normal surface $S$, furthermore $\kappa(X)=-\infty$ and $S \cdot C_{t} \geq 0$. In this case we expect that $X$ carries a "generic" conic bundle structure, in particular $X$ is uniruled and it is proved in $[23,3.5]$ that, given a generic conic bundle structure on $X$, then $X$ carries a contraction.

The aim of this section is to settle Case (E), so that we obtain:

Theorem 2.2. - Let $X$ be a smooth compact Kähler threefold, $C \subset X$ a rational curve with $K_{X} \cdot C<0$. Then there exists a surjective morphism $\varphi$ : $X \rightarrow Y$ to a normal compact complex space $Y$ such that

1) $\varphi$ has connected fibers;

2) $-K_{X}$ is $\varphi$-ample;

3) $\rho(X)=\rho(Y)+1$;

4) $Y$ is Kähler except possibly if $Y$ is smooth and $\varphi$ is blow-up along a smooth curve. In this last case $Y$ is Kähler if and only if the class of a fiber is extremal in the closure of the dual of the Kähler cone.

Proof. - As explained in 2.1, we have only to deal with the case (E). So assume that we are in the situation of $(\mathrm{E})$.

BULLETIN DE LA SOCIÉtÉ MATHÉMATIQUE DE FRANCE 
1) First we claim that it is sufficient to prove that $X$ is uniruled. In fact, if $X$ is uniruled, then [1,2.10] yields $a(X) \neq 2$ and the proof of ibid. (2.12) shows that $a(X) \neq 0$. Thus $a(X)=1$. Now [1, 2.13] shows that $X$ carries a generic conic bundle structure (we used in (2.13) the assumption of algebraic approximability only to conclude uniruledness!). Hence a contraction exists as explained in 2.1 .

2) In case $X$ is not simple, we can now just apply the main result of [2] to conclude from $\kappa(X)=-\infty$ that $X$ is uniruled. However our reasoning below for the "simple" case also applies here and makes the proof of (2.2) independent of [2]. In order to prove uniruledness we consider the normalisation $\nu: \tilde{S} \rightarrow S$. By $[1,1.3,2.5] \tilde{S}$ is a smooth minimal ruled surface, so we have a $\mathbb{P}_{1}$-bundle structure $g: \tilde{S} \rightarrow \tilde{C}$ over a smooth curve $\tilde{C}$. Let $N \subset S$ be the non-normal locus, equipped the conductor ideal and $\tilde{N} \subset \tilde{S}$ be the analytic preimage.

3) We shall assume here that there is a multisection $C_{1} \subset \tilde{N}$ of $g$ and a fiber $F$ of $g$ such that $\nu\left(C_{1}\right)=\nu(F)$. We shall use the standard theory of ruled surfaces as treated in $[9, \mathrm{~V} .2]$. Let $C_{0}$ be a section of $\tilde{S}$ with $C_{0}^{2}$ minimal and let $e=-C_{0}^{2}$. Then we can write

$$
C_{1}=a C_{0}+b F
$$

with $a \geq 1$ (here = means numerical equivalence). Consider the "cycle map" $\nu_{*}: N_{1}(\tilde{S}) \rightarrow N_{1}(X)$. I claim that for every class $C^{\prime} \in \overline{N E}(\tilde{S})$ there is a positive rational number $\mu$ such that

$$
\nu_{*}\left(C^{\prime}\right)=\mu C_{t}=\mu \nu_{*}(F) .
$$

By our assumption

$$
\nu_{*}\left(C_{1}\right)=\lambda \nu_{*}(F)
$$

with a positive rational number $\lambda$. Hence

$$
\nu_{*}\left(a C_{0}\right)=\nu_{*}\left(C_{1}\right)-b \nu_{*}(F)=(\lambda-b) \nu_{*}(F) .
$$

Let $\omega$ be a Kähler form on $X$. Then

$$
\int_{C_{0}} \nu^{*}(\omega)=\left(\frac{\lambda-b}{a}\right) \int_{C_{t}} \omega>0,
$$

hence $\lambda>b$ and our claim (*) holds for $C^{\prime}=C_{0}$. If now $e \geq 0$, then $\overline{N E}(\tilde{S})=$ $\mathbb{R}_{+}\left(C_{0}\right)+\mathbb{R}_{+}(F)$, and $(*)$ follows. If however $e<0$, then

$$
\overline{N E}(\tilde{S})=\mathbb{R}_{+} C^{\prime}+\mathbb{R}_{+} F
$$

with $C^{\prime}=C_{0}+\frac{1}{2} e F$. Thus

$$
\nu_{*}\left(C^{\prime}\right)=\nu_{*}\left(C_{0}\right)+\frac{e}{2} \nu_{*}(F)=\left(\frac{\lambda-b}{a}+\frac{e}{2}\right) \nu_{*}(F) .
$$

Integrating the Kähler form $\omega$ again, we see that the coefficient is positive, so we are done with $\left(^{*}\right)$. Our conclusion from $\left(^{*}\right)$ is the following:

TOME $129-2001-\mathrm{N}^{\mathrm{O}} 3$ 
$(* *)$ if $L$ is a line bundle on $S$ such that $L \cdot C_{t}>0\left(\right.$ resp. $\left.L \cdot C_{t}=0\right)$ then $\nu^{*}(L)$ is ample, so $L$ is ample (resp. $L \equiv 0$ ).

Since $S \cdot C_{t} \geq 0$, we deduce that either

(a) the normal bundle $N_{S}$ is ample, or

(b) $N_{S} \equiv 0$.

In case (a) we have $S^{3}>0$, hence $X$ is projective [1, 2.9]. In case (b) by [20], [26], we have $H^{1}\left(S, \mathcal{O}_{S}\right)=0$ since the anticanonical bundle $-K_{S}=\omega_{S}^{*}$ is ample. In particular

$$
\operatorname{Pic}(S) \subset H^{2}(S, \mathbb{Z})
$$

We claim that

$$
N_{S} \simeq \mathcal{O}_{S}
$$

In order to prove $(+)$ we will verify that $\tilde{N}$ consists only of $C_{0}$ and one fiber $F$ and that $C_{0} \simeq \mathbb{P}_{1}$ so that in particular

$$
H^{1}(\tilde{N}, \mathbb{Z})=0 .
$$

Assuming $(++)$ for the moment, we consider the Mayer-Vietoris type sequence

$$
H^{1}(\tilde{N}, \mathbb{Z}) \longrightarrow H^{2}(S, \mathbb{Z}) \longrightarrow H^{2}(\tilde{S}, \mathbb{Z}) \oplus H^{2}(N, \mathbb{Z}) \longrightarrow H^{2}(\tilde{N}, \mathbb{Z}) .
$$

Then $(++)$ implies

$$
H^{2}(S, \mathbb{Z}) \subset H^{2}(\tilde{S}, \mathbb{Z}) \oplus H^{2}(N, \mathbb{Z}) \simeq \mathbb{Z}^{3}
$$

(notice that the reduction of $N$ is an irreducible curve because $\tilde{N}$ has only two components). Thus $H^{2}(S, \mathbb{Z})$ is torsion free. Moreover $c_{1}\left(N_{S}\right)=0$ and therefore $(+)$ follows from $\operatorname{Pic}(S) \subset H^{2}(S, \mathbb{Z})$.

From $H^{1}\left(S, \mathcal{O}_{S}\right)=0$ and $H^{2}\left(S, \mathcal{O}_{S}\right)=H^{0}\left(S, \omega_{S}\right)=0\left(\right.$ note $\left.\omega_{S} \cdot C_{t}=-1\right)$, it now follows

$$
\chi\left(N_{S}\right)=\chi\left(\mathcal{O}_{S}\right)=1,
$$

hence $S$ deforms in a 1-dimensional family whose general member $S_{t}$ has again negative Kodaira dimension, i.e. $H^{0}\left(\omega_{S_{t}}^{m}\right)=0$ for all positive $m$ and therefore (going to a desingularisation) we see that $S_{t}$ is uniruled, hence $X$ is uniruled. It therefore remains to prove that $\tilde{N}$ has only two rational components in order to finish 3). Write

$$
\nu^{*}\left(\omega_{S}^{*}\right)=\alpha C_{0}+\beta F \quad \text { and } \quad \tilde{N}=\gamma C_{0}+\delta F .
$$

Then $\alpha=1$ (because $\nu^{*}\left(\omega_{S}^{*}\right) \cdot F=1$ ) and moreover $\gamma>0$ (because $\tilde{N}$ contains $C_{1}$ ), hence $\gamma \geq a$. We are going to use the equation

$$
\omega_{\tilde{S}}=\nu^{*}\left(\omega_{S}\right)-\tilde{N}
$$

(see [20], [26]). Since

$$
\omega_{\tilde{S}}^{*}=2 C_{0}+(e+2-2 b) F,
$$

BULletin DE LA SOCIÉtÉ MATHÉMATIQUE DE FRANCE 
$b$ the genus of $C_{0}$, i.e. the genus of $\tilde{C}$, we obtain

$$
2=\gamma+\alpha \text { and } e+2-2 b=\beta+\delta .
$$

Thus $\gamma=1$. Notice that $\delta>0$ since a fiber and a multi-section (now a section) are identified. Note also that $\nu^{*}\left(\omega_{S}^{*}\right)$ is ample by $\left(^{* *}\right)$. Now it is a simple calculation using [9, V.2] to obtain $b=0, e \geq 0, \beta=e+1$ and $\delta=1$. So $\tilde{N}=C_{0}+F$, even ideal-theoretically, with $C_{0} \simeq \mathbb{P}_{1}$. This proves $(++)$.

4) Finally we have to treat the case that no multisection of $g$ is identified with a fiber by $\nu$. Then $g$ induces a map $h: S \rightarrow B$ to a - usually non-normal - curve $B$ and a map $\mu: \tilde{C} \rightarrow B$, the normalisation of $B$, such that

$$
h \circ \nu=\mu \circ g .
$$

So for every $t \in T$ we find some $b \in B$ such that $C_{t} \subset S_{b}:=h^{-1}(b)$, and $S_{b}$ consists only of $C_{t}^{\prime} s$. We fix a general smooth point $b \in B$. Then $S_{b}$, the analytic fiber over $b$, is of the form

$$
S_{b}=C_{t_{1}}+\cdots+C_{t_{r}}
$$

and of course $S_{b}$ is as a Cartier divisor in a Gorenstein surface free from embedded points. Actually $S_{b}$ is locally a complete intersection (in $X$ ).

(a) If $S_{b}$ is reducible, then

$$
-K_{X} \cdot S_{b} \geq 2
$$

hence by Ein's deformation lemma (see [17, II.1.16]) the cycle $S_{b}$ of rational curves deforms in an at least 2-dimensional family. Since the deformations of $S_{b}$ inside $S$ have dimension 1 , we conclude the existence of a covering family $\left(B_{s}\right)$ of curves for $X$ (the general $B_{s}$ might however not be rational). So $X$ is not simple, the only case to be excluded if we use [2]. Here is the way to avoid [2]: let $u: \tilde{S}_{b}=\nu^{-1}\left(S_{b}\right) \rightarrow S_{b}$ be the normalisation of $S_{b}$. Then $\tilde{S}_{b}$ consists of $r$ disjoint smooth rational curves. Now

$$
\operatorname{dim}_{[u]} \operatorname{Hom}\left(\tilde{S}_{b}, X\right) \geq-K_{X} \cdot S_{b}+3 \chi\left(\mathcal{O}_{\tilde{S}_{b}}\right)=-K_{X} \cdot S_{b}+3 r,
$$

see e.g. $\quad\left[17\right.$, II.1.15]. Taking into account $\operatorname{dim} \operatorname{Aut}\left(\tilde{S}_{b}\right)=3 r$, we can still conclude the existence of a covering family of reducible rational curves for $X$ so that $X$ is uniruled.

(b) Suppose finally that $S_{b}$ is irreducible. Having in mind that $S_{b}$ is Cartier in $S$ and also locally a complete intersection, we consider the exact sequence of conormal bundles

$$
0 \rightarrow N_{S \mid X}^{*} \mid S_{b} \longrightarrow N_{S_{b} \mid X}^{*} \longrightarrow N_{S_{b} \mid S}^{*} \rightarrow 0 .
$$

Since $N_{S_{b} \mid S}^{*}=\mathcal{O}_{S_{b}}$ and since $N_{S \mid X}^{*} \mid S_{b}$ has nef dual, it follows via the sequence that $N_{S_{b} \mid X}$ is nef. This contradicts easily $K_{X} \cdot C_{t}=-1$.

TOME $129-2001-\mathrm{N}^{\mathrm{O}} 3$ 


\section{Existence of rational curves}

In this section we prove that any smooth compact Kähler threefold $X$ whose canonical bundle is not nef, carries a rational curve $C$ with $K_{X} \cdot C<0$, unless $X$ is simple with negative Kodaira dimension. We first treat the case $\kappa(X) \geq 0$.

TheOREM 3.1. - Let $X$ be a smooth compact Kähler threefold with $\kappa(X) \geq 0$. If $K_{X}$ is not nef, there exists a rational curve $C \subset X$ with $K_{X} \cdot C<0$, and therefore there is a contraction $\varphi: X \rightarrow Y$.

Proof. - Since $\kappa(X) \geq 0$, we find $m \in \mathbb{N}$ such that

$$
m K_{X}=\mathcal{O}_{X}\left(\sum \lambda_{i} D_{i}\right),
$$

where $\lambda_{i} \in \mathbb{N}$ and $D_{i} \subset X$ are irreducible. By [23, 4.9] there exists some irreducible component $D_{i_{0}}$ such that $K_{X} \mid D_{i_{0}}$ is not nef. By renumbering we may assume that the components with this property are exactly $D_{1}, \ldots, D_{r}$. Fix some $1 \leq i \leq r$ and put $D=D_{i}$. Let $\nu: \tilde{D} \rightarrow D$ be the normalisation and $\pi: \hat{D} \rightarrow \tilde{D}$ the minimal desingularisation. From adjunction we immediately obtain that

$$
K_{D}=r K_{X} \mid D-E,
$$

where $r>1$ is a positive rational number and $E$ an effective $\mathbb{Q}$-divisor. Next observe that

$$
K_{\hat{D}}=\pi^{*} \nu^{*}\left(K_{D}\right)-E^{\prime}
$$

with another effective divisor, so that, putting $L=\pi^{*} \nu^{*}\left(K_{X} \mid D\right)$, we obtain a formula of $\mathbb{Q}$-divisors

$$
L=a K_{\hat{D}}+A
$$

with a positive rational number $a<1$ and an effective $\mathbb{Q}$-divisor $A$.

1) In a first step we construct an irreducible curve $C \subset D$ such that $K_{X} \cdot C<0$. If $a(D)=2$, the existence of $C$ is clear, $K_{X} \mid D$ being not nef.

1.1) Suppose next $a(D)=0$. Then $\hat{D}$ is bimeromorphically a K3-surface or a torus. Then by $\left(^{* *}\right), L$ has to be effective : there are curves $C_{i} \subset \hat{D}$ and positive integers $p$ and $m_{i}$ such that

$$
p L=\sum m_{i} C_{i} .
$$

Observe that every curve in $\hat{D}$ is rational! So by $[23,4.9]$ we obtain a rational curve $C \subset \hat{D}$ with $L \cdot C<0$, hence $K_{X} \cdot \nu(\pi(C))<0$. Thus in case $a(D)=0$ for some component $D$ we are already settled with the theorem.

1.2) Now suppose $a(D)=1$ and let $f: \hat{D} \rightarrow B$ be the algebraic reduction, an elliptic fibration. Since $L$ is not nef, $[23,4.13]$ implies that either there exists an irreducible curve $C \subset \hat{D}$ with $L \cdot C<0$ or $L=f^{*}(G)$ with $G^{*}$ ample on $B$. This second alternative is clearly impossible since $L$ is effective. Hence 1 ) holds also in this case.

BULLETIN DE LA SOCIÉtÉ MATHÉMATIQUE DE FRANCE 
2) In all what follows we may assume $C$ irrational, otherwise we are done. Now $C$ deforms by [17, II.1.16] in an at least 1-dimensional family in $X$, therefore we can "extract" a maximal non-splitting family $\left(C_{t}\right)$ of irreducible curves such that $K_{X} \cdot C_{t}<0$. Here "maximal" means that no deformation of the general $C_{t}$ splits. In other words, we can choose $T$ to be (or rather project to) an irreducible component of the cycle space (which is automatically compact, $X$ being Kähler). By $K_{X} \cdot C_{t}<0$ all the $C_{t}$ have to be in one of the $D_{j}^{\prime} s$ for $1 \leq j \leq r$, say in $D$. By $\left({ }^{* *}\right)$, we have $K_{D} \cdot C_{t}<0$. Let $\hat{C}_{t}$ be the strict transform of a general $C_{t}$ in $\hat{D}$. Then

$$
L \cdot \hat{C}_{t}<0
$$

In particular (**) yields $K_{\hat{D}} \cdot \hat{C}_{t}<0$. Then, $\hat{C}_{t}$ being irrational, $\hat{C}_{t}$ deforms in an at least 1-dimensional family, see [17, II.1.14, 1.15], and we can either extract a maximal non-splitting family $\left(B_{s}\right)$ of irrational curves on $\hat{D}$ such that

$$
L \cdot B_{s}<0
$$

or we obtain the rational curve in $D$ we are looking for. To be more precise, assume that we have a splitting. Take a splitting component $C^{\prime}$ with $L \cdot C^{\prime}<0$. If $C^{\prime}$ is irrational, then $C^{\prime}$ deforms in a family and we proceed as before (inductively). If $C^{\prime}$ is rational, then its image $C^{\prime \prime}$ in $D$ is a rational curve with $K_{X} \cdot C^{\prime \prime}<0$ are we are done. Notice however that on surfaces $X$ it is sometimes not possible to extract a non-splitting family from a splitting family $\left(C_{t}\right)$ with $K_{X} \cdot C_{t}<0$. The reason is that there are curves with $K_{X} \cdot C<0$ which do not move, namely $(-1)$-curves, and these are the only ones.

Now we pass to a minimal model $\sigma: \hat{D} \rightarrow D_{0}$ and consider the induced family $\left(B_{s}^{\prime}\right)$. Since $\hat{D}$ contains a covering family, $\left(\hat{C}_{t}\right)$, with $K_{\hat{D}} \cdot \hat{C}_{t}<0$, we have $\kappa(\hat{D})=-\infty$. So $D_{0}$ is a ruled surface or $\mathbb{P}_{2}$. Clearly $\left(B_{s}^{\prime}\right)$ is a non-splitting family.

Suppose first that $\hat{D}$ itself is not minimal, i.e. $\sigma$ is not an isomorphism. Then we may assume that $D_{0}$ is not the projective plane, otherwise we just omit the first blow up and substitute $D_{0}$ by the ruled surface $\mathbb{P}\left(\mathcal{O}_{\mathbb{P}_{1}} \oplus \mathcal{O}_{\mathbb{P}_{1}}(-1)\right)$ and argue with that ruled surface. Let $p \in D_{0}$ be a point blown up by $\sigma$. Then $p \in B_{s}^{\prime}$ for some $s$. Since $\left(B_{s}\right)$ is non-splitting, we must have $p \in B_{s}^{\prime}$ for all $s$. Then Lemma 3.3 gives a contradiction.

So $\hat{D}$ must be minimal. If $\hat{D}$ is the projective plane, then clearly $\left(B_{s}\right)$ has to be a family of lines. Anyway, then $\hat{D}=\tilde{D}$ and $L^{*}$ is ample in that case, so the existence of $C$ rational with $K_{X} \cdot C<0$ is clear. So $\hat{D}$ is ruled with ruling $p: \hat{D} \rightarrow B$. By Lemma 3.4, its invariant $e<0$, in particular $\hat{D}$ has no exceptional curves and $\tilde{D}=\hat{D}$. Let $F$ be a fiber of $p$ and $C_{0}$ be a section with $C_{0}^{2}=-e$ minimal. Let $Z$ denote a class in the boundary component of $\overline{N E}(\hat{D})$

TOME $129-2001-\mathrm{N}^{\mathrm{O}} 3$ 
different from the ray generated by $F$. Then after rescaling

$$
Z \equiv C_{0}+\frac{e}{2} F
$$

Since $K_{\hat{D}} \cdot Z \geq 0$, we conclude from (**) easily that

$$
L \cdot Z \geq 0
$$

(consider curves near to the ray $\mathbb{R}_{+}(Z)$.) Using our assumption $L \cdot F \geq 0$ (otherwise we are done), we conclude that $L$ is nef, contradicting $L \cdot \hat{C}_{t}<0$.

Notice that in the second part of the proof of 3.1, where we deduced the existence of rational curve $C \subset X$ with $K_{X} \cdot C<0$ from the existence of some curve $C$ with $K_{X} \cdot C<0$ we did not use the assumption $\kappa(X) \geq 0$. Therefore we have

Theorem 3.2. - Let $X$ be a smooth compact Kähler threefold. Assume that there is an irreducible curve $C \subset X$ with $K_{X} \cdot C<0$. Then there is a rational curve $C \subset X$ with $K_{X} \cdot C<0$ and hence there is a contraction on $X$.

We still have to prove the following two lemmata.

LEMma 3.3. - Let $S$ be a ruled surface, $p \in S$. Let $\left(C_{t}\right)_{t \in T}$ be family of curves with $C_{t}$ irreducible for general t and $T$ compact (as always). Assume that $p \in C_{t}$ for all $t$. Then $\left(C_{t}\right)$ splits.

Proof. - Assume that $\left(C_{t}\right)$ does not split. Of course we may restrict ourselves to the case $\operatorname{dim} T=1$. Let $f: S \rightarrow B$ be a ruling, $F_{b}$ the fiber over $b \in B$. Since $C_{t}$ cannot be a fiber, it must be a multi-section of $f$ of degree $d$ and therefore $C_{t} \cap F_{b}$ consists of $d$ points, counted with multiplicity. The case $d=1$ is trivially excluded, e.g. by considering the graph of the family $\left(C_{t}\right)$ which is a product. Denoting by $\operatorname{Hilb}_{d}(X)$ the Hilbert scheme of $d$ points in a projective manifold $X$, we obtain for every $b$ a map

$$
\varphi_{b}: T \longrightarrow \operatorname{Hilb}_{d}\left(F_{b}\right) .
$$

Let $b_{0}=f(p)$. Choose an open neighborhood $U$ of $b_{0}$ in $B$ and a trivialisation $S_{U} \simeq U \times \mathbb{P}_{1}$. Then we obtain a map

$$
\Phi: U \times T \longrightarrow \operatorname{Hilb}_{d}\left(\mathbb{P}_{1}\right)
$$

such that (by abuse of notation) $\Phi \mid\{b\} \times T=\varphi_{b}$. Now notice that $\operatorname{Hilb}_{d}\left(\mathbb{P}_{1}\right)=$ $S^{d} \mathbb{P}_{1}$, the $d$-th symmetric product of $\mathbb{P}_{1}$. Since all $C_{t}$ pass through $p$, we conclude

$$
\varphi_{b_{0}}(T) \subset\{p\} \times S^{d-1} \mathbb{P}_{1} \subset S^{d} \mathbb{P}_{1} .
$$

Using the identification $S^{d} \mathbb{P}_{1}=\mathbb{P}_{d}$, the curve $\varphi_{b_{0}}(T)$ is contained in a hyperplane, i.e. degenerates. After shrinking $U$, there is no point $q \in F_{b}, b \in U$, $b \neq b_{0}$ such that all $C_{t}$ pass through $q$. Therefore $\varphi_{b}(T)$ is not degenerate for $b \in U, b \neq b_{0}$. This leads to a contradiction since the $\varphi_{b}(T)$ form a family 
of curves: first if $d=2$, then $\varphi_{b_{0}}(T)$ is a line, whereas $\varphi_{b}(T)$ is not for $b \neq b_{0}$; if $d \geq 3$, choose a generic projection $g: \mathbb{P}_{d} \rightarrow \mathbb{P}_{2}$ and consider the induced holomorphic maps $g \circ \varphi_{b}$.

LEMma 3.4. - Let $S$ be a ruled surface, $\left(C_{t}\right)_{t \in T}$ be a maximal (i.e. $T$ projects to an irreducible component of the cycle space) non-splitting family of curves not consisting of fibers such that $K_{S} \cdot C_{t}<0$. Then $S$ comes from a stable vector bundle, i.e. its invariant $e$ is negative.

Proof. - We write for numerical equivalence

$$
C_{t} \equiv k C_{0}+b F
$$

where $C_{0}$ is a section with minimal $C_{0}^{2}$ and $F$ is a fiber of $f$, a ruling. Again let $e=-C_{0}^{2}$. More precisely we write

$$
\mathcal{O}_{S}\left(C_{t}\right)=\mathcal{O}_{S}\left(C_{0}\right) \otimes f^{*}\left(G_{t}\right)
$$

with a line bundle $G_{t}$ of degree $b$ on $B$. By virtue of

$$
K_{S}=-2 C_{0}+(2 g-2-e) F
$$

we obtain

$$
K_{S} \cdot C_{t}=2 k e+k(2 g-2-e)-2 b .
$$

Hence $K_{S} \cdot C_{t}<0$ translates into

$$
b>\frac{k e}{2}+k(g-1) .
$$

If now $e \geq 0$, i.e. $E$ is not stable, then $\left(^{*}\right)$ implies $b>g-1$, hence RiemannRoch gives $h^{0}\left(G_{t}\right)>0$ and therefore we obtain a splitting inside the family.

We now turn to the case $\kappa(X)=-\infty$.

TheOREm 3.5. - Let $X$ be a smooth compact Kähler threefold or a normal compact Kähler threefold which is $\mathbb{Q}$-factorial with at most terminal singularities. Assume $\kappa(X)=-\infty$ and $X$ not simple. Then there exists a rational curve $C \subset X$ with $K_{X} \cdot C<0$.

Proof. - Of course we have only to treat the non-projective case. By [2], $X$ is uniruled. Hence our claim is clear, taking $C$ to be a general member of a covering family of rational curves of $X$.

REMARKs 3.6. - 1) In order to complete the picture that a compact Kähler threefold $X$ with $K_{X}$ not nef carries a rational curve $C$ with $K_{X} \cdot C<0$ it remains to prove that a simple Kähler threefold with $\kappa(X)=-\infty$ carries some curve (not necessarily rational) with $K_{X} \cdot C<0$. Of course this is a strange situation, which is expected not to exist. In 2) and 3) we treat some special cases.

2) There is no simple compact Kähler manifold $X$ with $\kappa(X)=-\infty$ such that $-K_{X}$ is hermitian semi-positive. In fact if $X$ is simple, then

TOME $129-2001-\mathrm{N}^{\mathrm{O}} 3$ 
$q(X)=0$ and the same holds for every finite étale cover of $X$, hence [5] implies $H^{2}\left(X, \mathcal{O}_{X}\right)=0$, contradiction.

3) The same should be true if $-K_{X}$ is only nef. We may clearly assume $q(X)=0$. Since

$$
\chi\left(X,-m K_{X}\right)=(2 m+1) \chi\left(X, \mathcal{O}_{X}\right)
$$

(Riemann-Roch; observe $K_{X}^{3}=0$, since otherwise $X$ is projective), we obtain a contradiction if we can prove that

$$
h^{2}\left(-m K_{X}\right) \leq C
$$

for a positive constant $C$ and all positive integers $m$, at least if $m$ is sufficiently divisible.

We finally summarize the results of this section:

THEOREM 3.7. - Let $X$ be a smooth compact Kähler threefold with $K_{X}$ not nef. Then there exists a rational curve $C \subset X$ with $K_{X} \cdot C<0$ and therefore $X$ carries a contraction unless (possibly) $X$ is simple with $\kappa(X)=-\infty$.

\section{Kähler contact threefolds}

Let $X$ be a smooth compact Kähler threefold. We recall the notion of a contact structure on $X$ and refer to [18] for details. Assume that there is a line bundle $L$ with $-K_{X}=2 L$. A contact structure on $X$ is an $L$-valued 1-form

$$
\omega \in H^{0}\left(\Omega_{X}^{1} \otimes L\right)
$$

without zeroes such that locally $\omega \wedge d \omega$ is nowhere 0 . We therefore have a vector bundle $F$ of rank 2 and an exact sequence

$$
0 \rightarrow F \longrightarrow T_{X} \longrightarrow L \rightarrow 0 .
$$

Ye $[29]$ proved that a projective contact threefold is either $\mathbb{P}_{3}$ or the projectivised tangent bundle of any projective surface. We extend this results to the Kähler case; however we cannot handle at the moment the possibility of a simple contact threefold with negative Kodaira dimension.

THEOREM 4.1. - Let $X$ be a smooth compact Kähler threefold admitting a contact structure. Then $X$ is one of the following

1) $X=\mathbb{P}_{3}$;

2) $X$ is the projectivised tangent bundle of a compact Kähler surface;

3) $X$ is simple with $\kappa(X)=-\infty$.

Of course case 3 ) should not exist. Note also that the projectivised tangent bundle of a Kähler surface has always a contact structure, see e.g. [18]. 
Proof. - The projective case being proved in [29], we shall assume $X$ not projective. We may also assume that $X$ is not simple with $\kappa(X)=-\infty$.

1) First notice that the arguments in [29] show that $\kappa(X) \leq 0$. In fact, the necessary results of Bogomolov are true in the Kähler case, too.

2) Suppose that $K_{X}$ is not nef. Then by (3.7) we find a contraction $\varphi: X \rightarrow Y$. Since $K_{X}$ is divisible by $2, \varphi$ must be the blow-up of a smooth point or (analytically) a $\mathbb{P}_{1}$-bundle over a Kähler surface, cp. [29]. Assume first that $\varphi$ is the blow-up of a point with exceptional divisor $E$. Using the notations from the beginning of the section, we have an exact sequence

$$
0 \rightarrow N_{E}^{*} \otimes L \longrightarrow \Omega_{X}^{1} \otimes L\left|E \longrightarrow \Omega_{E}^{1} \otimes L\right| E \rightarrow 0 .
$$

Now $L \mid E=\mathcal{O}_{E}(1)$, hence

$$
H^{0}\left(\Omega_{X}^{1} \otimes L \mid E\right)=H^{0}\left(N_{E}^{*} \otimes L\right) .
$$

But since $N_{E}^{*} \otimes L=\mathcal{O}(2)$, every section in $\Omega_{X}^{1} \otimes L$ must have zeroes, contradiction. Hence $\varphi$ is a $\mathbb{P}_{1}$-bundle over a Kähler surface $Y$ and it follows as in [29] that $X$ is actually $\mathbb{P}\left(T_{Y}\right)$.

3) It remains to treat the case that $K_{X}$ is nef and $\kappa(X)=0$. We could apply [3], but here we give a simple direct argument. If $X$ is not simple, then $K_{X} \equiv 0$ by 1.1 . Now a finite étale cover of $X$ is either a torus or a product of a K3-surface with an elliptic curve which immediately gives a contradiction. So we will suppose that $X$ is simple. The contact form is a section in the group $H^{0}\left(X, \Omega_{X}^{1} \otimes L\right)$ with $-K_{X}=2 L$. Hence we have an injection $L^{*} \rightarrow \Omega_{X}^{1}$. If we knew that $H^{0}\left(L^{*}\right) \neq 0$ then $q(X) \geq 1$ and $X$ is a torus (see $1.1,1.4$ ). However in general we know only that $\kappa\left(L^{*}\right)=\kappa(X)=0$, i.e. $H^{0}\left(L^{* m}\right) \neq 0$ for some positive $m$. Then we take a generically finite map $f: \tilde{X} \rightarrow X$ such that

$$
H^{0}\left(\tilde{X}, f^{*}\left(L^{*}\right)\right) \neq 0
$$

and $\tilde{X}$ is again smooth. Since $f^{*}\left(L^{*}\right) \subset f^{*}\left(\Omega_{X}^{1}\right)$ we obtain via the canonical injective $\left(X\right.$ is smooth!) map $f^{*}\left(\Omega_{X}^{1}\right) \rightarrow \Omega_{\tilde{X}}^{1}$ an injection

$$
f^{*}\left(L^{*}\right) \subset \Omega_{\tilde{X}}^{1}
$$

and therefore $q(\tilde{X})>0$. Now $\tilde{X}$ must again be simple, in particular $\kappa(\tilde{X})=0$. Hence $\tilde{X}$ is Kummer, more precisely the Albanese map $\alpha: \tilde{X} \rightarrow A$ is bimeromorphic onto the threedimensional torus $A$. Therefore $K_{\tilde{X}}=B$ with $B$ an effective divisor supported on the exceptional locus of $\alpha$. On the other hand

$$
K_{\tilde{X}}=f^{*}\left(K_{X}\right)+R
$$

with another effective divisor $R$. Since $K_{X}$ is nef, it follows easily that $R=B$ and thus $f^{*}\left(K_{X}\right)=\mathcal{O}_{\tilde{X}}$, i.e. $K_{X} \equiv 0$, or directly a covering $A \rightarrow X$. So $X$ is Kummer. 
Remark 4.2. - Assume that $X$ is a simple threefold, $\kappa(X)=-\infty$ and $X$ admits a contact structure. If there is a curve $C$ with $K_{X} \cdot C<0$, then by 3.2 we have a contraction $\varphi: X \rightarrow Y$. This is ruled out as in the proof of 4.1. Hence we can at least say that $K_{X} \cdot C \geq 0$ for all curves $C \subset X$.

REMARK 4.3. - Observe that we have proved in 4.1 the following slightly more general statement.

Let $X$ be a smooth compact Kähler threefold, $L \in \operatorname{Pic}(X)$ such that $-K_{X}=2 L$. Let $\omega \in H^{0}\left(\Omega_{X}^{1} \otimes L\right)$ be a section without zeroes. Then $X$ is one the following

(a) $X=\mathbb{P}_{3}$;

(b) $X=\mathbb{P}\left(T_{S}\right)$, where $S$ is a Kähler surface;

(c) $X$ is an étale "undercover" of a torus or a product of an elliptic curve with a K3-surface;

(d) $X$ is simple.

We refer to [15] for recent results on higher dimensional contact manifolds.

\section{BIBLIOGRAPHY}

[1] Campana (F.) \& Peternell (T.) - Towards a Mori theory on compact Kähler threefolds, I, Math. Nachr., t. 187 (1997), pp. 29-59.

[2] Complex threefolds with non-trivial holomorphic 2-forms, J. Alg. Geom., t. 9 (2000), pp. 223-264.

[3] Demailly (J.-P.) - Frobenius integrability of certain holomorphic $p$ forms, Preprint, 2000, to appear in a volume in honour of H. Grauert.

[4] Demailly (J.-P.), Peternell (T.) \& Schneider (M.) - Compact complex manifolds with numerically effective tangent bundles, J. Alg. Geom., t. 3 (1994), pp. 295-345.

[5] Compact Kähler manifolds with hermitian semipositive anticanonical bundle, Comp. Math., t. 101 (1996), pp. 217-224.

[6] FuJiki (A.) - On the structure of compact complex manifolds in class $\mathcal{C}$, Adv. Stud. Pure Math., vol. 1, 1983, pp. 231-302.

[7] Fujita (T.) - Kähler fiber spaces over curves, J. Math. Soc. Japan, t. 30 (1978), pp. 779-794.

[8] Hanamura (M.) - On the birational automorphism groups of algebraic varieties, Comp. Math., t. 63 (1987), pp. 123-142.

[9] Hartshorne (R.) - Algebraic geometry, Graduate Texts in Math., vol. 52, Springer, 1977.

[10] Kawamata (Y.) - Characterisation of abelian varieties, Comp. Math., t. 43 (1981), pp. 253-276.

BULletin DE LA SOCIÉtÉ MATHÉMATIQUE DE FRANCE 
[11] , Pluricanonical systems on minimal algebraic varieties, Inv. Math., t. 79 (1985), pp. 567-588.

[12] Crepant blowing ups of threedimensional canonical singularities and applications to degenerations of surfaces, Ann. Math., t. 119 (1988), pp. 603-633.

[13] _ Abundance theorem for minimal threefolds, Inv. Math., t. 108 (1992), pp. 229-246.

[14] Kawamata (Y.) \& Viehweg (E.) - On a characterisation of an abelian variety in the classification theory of algebraic varieties, Comp. Math., t. 41 (1980), pp. 355-359.

[15] Kebekus (S.), Peternell (T.), Sommese (A.J.) \& Wisniewski (J.) - Projective contact manifolds, Inv. Math., t. 142 (2000), pp. 1-15.

[16] Kollár (J.) - Flops, Nagoya Math. J., t. 113 (1989), pp. 15-36.

[17] _ Rational curves on algebraic varieties, Erg. d. Math., vol. 32, Springer, 1996.

[18] LeBrun (C.) - Fano manifolds, contact structures and quaternionic geometry, Int. J. Math., t. 6 (1995), pp. 419-437.

[19] Miyaoka (Y.) - Abundance conjecture for threefolds: $\nu=1$ case, Comp. Math., t. 68 (1988), pp. 203-220.

[20] Mori (S.) - Threefolds whose canonical bundles are not numerically effective, Ann. Math., t. 116 (1982), pp. 133-176.

[21] , Flip theorem and the existence of minimal models for 3-folds, J. Amer. Math. Soc., t. 1 (1988), pp. 117-253.

[22] NakAyama (N.) - The lower semi-continuity of the plurigenera of complex varieties, Adv. Stud. Pure Math., vol. 10, 1987, pp. 551-590.

[23] Peternell (T.) - Towards a Mori theory on compact Kähler threefolds, II, Math. Ann., t. 311 (1998), pp. 729-764.

[24] ReID (M.) - Canonical threefolds, in Géométrie algébrique, Angers, vol. 1, Sijthoff and Noordhoff, pp. 273-310.

[25] Minimal models of threefolds, Adv. Stud. Pure Math., vol. 1, 1983, pp. 131-180.

[26] _ Singular del Pezzo surfaces, Publ. RIMS, t. 30 (1994), pp. 695728.

[27] Ueno (K.) - On compact analytic threefolds with non-trivial Albanese torus, Math. Ann., t. 278 (1987), pp. 41-70.

[28] VieHWEG (E.) - Klassifikationstheorie algebraischer Varietäten der Dimension 3, Comp. Math., t. 41 (1980), pp. 361-400.

[29] YE (Y.G.) - A note on complex projective threefolds admitting holomorphic contact structures, Inv. Math., t. 115 (1994), pp. 311-314. 